Article

\title{
Tobramycin Promotes Melanogenesis by Upregulating p38 MAPK Protein Phosphorylation in B16F10 Melanoma Cells
}

\author{
Seung-Hyun Moon, You Chul Chung and Chang-Gu Hyun * \\ Department of Chemistry and Cosmetics, Jeju National University, Jeju 63243, Korea \\ * Correspondence: cghyun@jejunu.ac.kr; Tel.: +82-64-754-3542
}

Received: 26 July 2019; Accepted: 2 September 2019; Published: 5 September 2019

check for updates

\begin{abstract}
Tobramycin is an aminoglycoside-based natural antibiotic derived from Streptomyces tenebrarius, which is primarily used for Gram-negative bacterial infection treatment. Although tobramycin has been utilized in clinical practice for a long time, it has exhibited several side effects, leading to the introduction of more effective antibiotics. Therefore, we conducted our experiments focusing on new possibilities for the clinical use of tobramycin. How tobramycin affects skin melanin formation is unknown. This study used B16F10 melanoma cells to assess the effect of tobramycin on melanin production. After cytotoxicity was assessed by MTT assay, melanin content and tyrosinase activity analyses revealed that tobramycin induces melanin synthesis in B16F10 cells. Next, Western blot analyses were performed to elucidate the mechanism by which tobramycin increases melanin production; phosphorylated p38 protein expression was upregulated. Protein inhibitors have been used to elucidate the mechanism of tobramycin. Kanamycin A and B are structurally similar to tobramycin, and 2-DOS represents the central structure of these antibiotics. The effects of these substances on melanogenesis were evaluated. Kanamycin A reduced melanin production, whereas kanamycin B and 2-DOS had no effect. Overall, our data indicated that tobramycin increases melanin production by promoting $\mathrm{p} 38$ protein phosphorylation in B16F10 melanoma cells.
\end{abstract}

Keywords: melanogenesis; B16F10 melanoma cell; aminoglycoside; tobramycin; melanin; p-p38; kanamycin; 2-DOS

\section{Introduction}

Ultraviolet radiation (UVR) is a mutagen and a nonspecific damaging agent, classified as a complete carcinogen, since it exhibits both tumor initiator and tumor promoter properties. UVR also causes various acute and chronic skin reactions. Acute human skin reactions to UVR include photodamage, erythema, mutation, and immunosuppression [1,2].

Melanin is produced by the melanocytes present in the skin to prevent UV damage. First, UV-mediated DNA damage in keratinocytes activates p53 and binds to and activates the pro-opiomelanocortin (POMC) gene. POMC polypeptides are post-translationally cleaved to produce adrenocortical stimulating hormones, $\alpha$-melanocyte-stimulating hormones $(\alpha-\mathrm{MSH})$, and $\beta$-endorphins. The secreted keratinocyte-derived $\alpha$-MSH signals to melanocytes through the G protein-coupled receptor melanocortin 1 receptor (MC1R) [3]. $\alpha$-MSH binds to the melanocyte MC1R and stimulates the adenylyl cyclase (AC) to increase the intracellular concentration of the secondary messenger cyclic adenosine monophosphate (cAMP). cAMP activates protein kinase A (PKA) and phosphorylates the cAMP response element (CREB) protein. In turn, CREB acts as a transcription factor for several genes, including the microphthalmia-associated transcription factor (MITF), a major regulator of melanogenesis. MITF binds to the M-box and regulates the expression of the melanin-producing enzymes tyrosinase (TYR), tyrosinase related protein 1 (TRP-1), and tyrosinase related protein 2 (TRP-2). 
Recent studies have revealed signaling pathways affecting the formation of various melanins, such as the phosphoinositide 3-kinase (PI3K)/protein kinase B (AKT), Wnt/ $\beta$-catenin, and mitogen-activated protein kinases (MAPK) signaling pathways; the MAPK pathway includes the extracellular signal-regulated kinase (ERK), c-Jun N-terminal kinase (JNK), and p38. It is also known that diverse factors, such as SCF/c-kit, nitric oxide (NO), and cytokines, inhibit or promote melanogenesis [3-5].

TYR and TRP-1,2 proteins migrate to the organelles called melanosomes, which produce two types of melanin (eumelanin and pheomelanin) [6]. Thus, melanin is formed in the skin, hair, and eyes; it not only protects the skin from UV, but also determines skin color [7,8]. Melanin, especially eumelanin, exerts a shielding effect and acts as a physical barrier scattering UVR and as an absorbent filter reducing UV penetration into the epidermis. Melanin efficacy as a sunscreen is assumed to be about 1.5-2.0 sun protection factor (SPF), and can reach up to $4 \mathrm{SPF}$, meaning that melanin absorbs $50 \%$ to $75 \%$ of the UVR [9].

Hypopigmentation in the skin is the result of melanin production reduction. This is due to a decrease in the amino acid tyrosine caused by melanin cell number reduction, melanin deficiency, or defects in melanin use by melanocytes. Common genetic causes of hypopigmentation include mutations in the tyrosinase gene. Symptoms encompass vitiligo, piebaldism, and albinism; various studies are under way to identify improved treatment options [10-12].

Tobramycin (Figure 1) is an aminoglycoside antibiotic with broad antibacterial spectrum in vitro, which is primarily used for treating serious Gram-negative bacterial infections [13]. Tobramycin is a natural compound, produced in culture broth of Streptomyces tenebrarius, and it is composed of a 2,6-diamino-2,6-dideoxy sugar and a 3-amino-3-deoxyglucose linked to the 4- and 6-hydroxyl groups of 2-deoxystreptamine [14,15]. It was discovered in 1967 and is currently still in clinical use [16-19]. However, tobramycin exhibits side effects such as kidney damage, hearing damage, and serious allergic reactions [15,20-22]. Over time, its use has gradually decreased due to the emergence of antibiotic-resistant bacteria and the development of more effective antibiotics [23-25]. Therefore, we initiated the current study to identify other possible clinical uses of tobramycin.

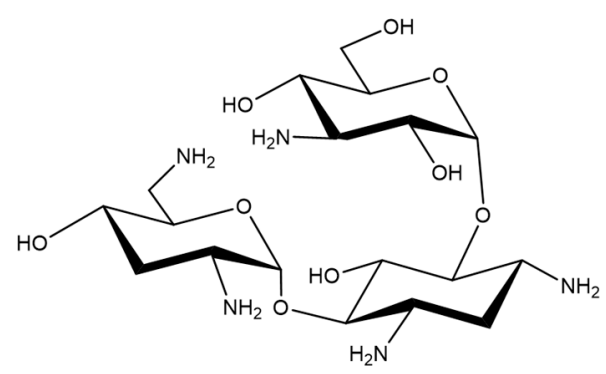

Figure 1. Chemical structure of Tobramycin.

\section{Results}

\subsection{Cell Viability of B16F10 Melanoma Cells}

The 3-(4,5-dimethylthiazol-2-yl)-2,5-diphenyltetrazolium bromide (MTT) assay was conducted to determine tobramycin cytotoxicity on B16F10 cells. The cells were cultured for $24 \mathrm{~h}$, treated with various concentrations of tobramycin, cultured for further $72 \mathrm{~h}$, and supplemented with the MTT reagent for cell viability measurement. Cytotoxicity was observed at $8 \mathrm{mg} / \mathrm{mL}$ concentration of tobramycin, and further experiments proceeded at a concentration of $4 \mathrm{mg} / \mathrm{mL}$ of tobramycin.

To evaluate if the selected concentrations of tobramycin could cause cellular damage and cytolysis, the lactate dehydrogenase $(\mathrm{LDH})$ released to the extracellular medium was spectrophotometrically quantified. It was observed that tobramycin 8 and $16 \mathrm{mg} / \mathrm{mL}$ induced LDH release $(60 \%$ and $400 \%$ variation, respectively, to untreated cell), while other concentrations of tobramycin did not alter LDH release relative to the untreated cell. Due to cellular toxicity induced by the high concentrations of tobramycin ( 8 and $16 \mathrm{mg} / \mathrm{mL}$ ), these concentrations were not considered for the present study (Figure 2). 


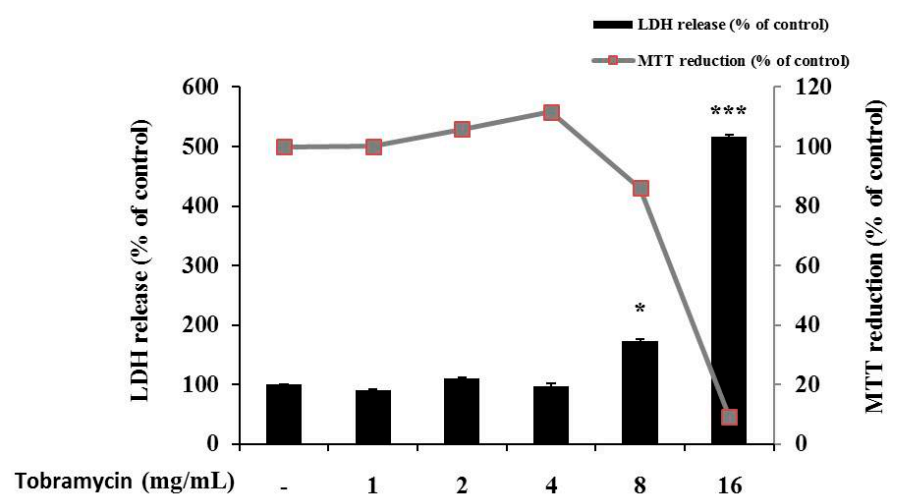

Figure 2. Cell viability of tobramycin-treated B16F10 melanoma cells. The cells were supplemented with various concentrations of tobramycin for $72 \mathrm{~h}$. Data are presented as mean \pm standard deviation (SD) of at least four independent experiments $(n=4)$. ${ }^{* *}$ indicates $p<0.01,{ }^{* * *} p<0.001$ vs. untreated cells.

\subsection{Effect of Tobramycin on Melanin Synthesis}

Melanin protein levels, produced in B16F10 cells after treatment with tobramycin at various concentrations $(0.5,1,2$, and $4 \mathrm{mg} / \mathrm{mL})$, were measured spectrophotometrically. In the cells treated with tobramycin for $72 \mathrm{~h}$, melanin production was increased in a concentration-dependent manner (Figure 3a). Figure 3b shows melanin accumulation in the cells; as the concentration of tobramycin increases, the melanin synthesis becomes macroscopically detectable.

(a)

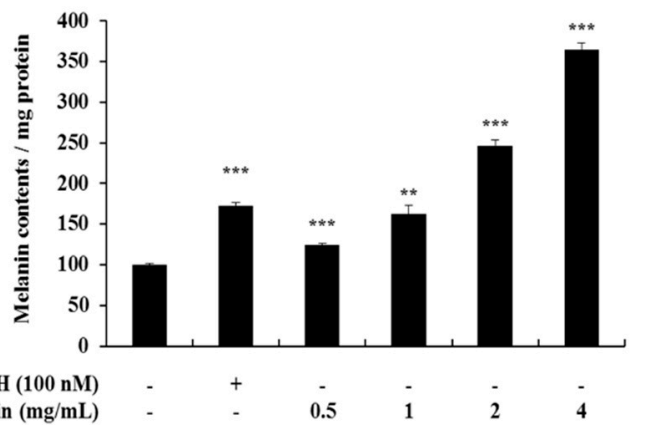

(b)

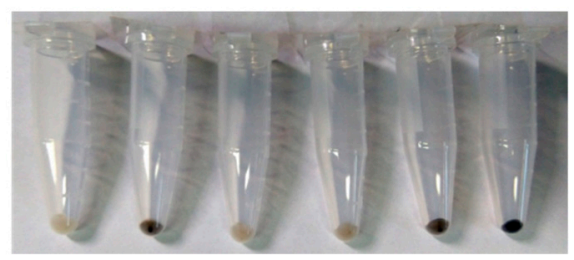

Figure 3. Melanin levels in tobramycin-treated B16F10 melanoma cells. The cells were treated with various concentrations of tobramycin for $72 \mathrm{~h}$, and $\alpha-\mathrm{MSH}$ was used as a positive control. (a) Melanin concentrations are expressed as percentages compared to the respective values obtained for the control cells. (b) Images of corresponding B16F10 cell pellets harvested by centrifugation. Data are presented as mean \pm standard deviation (SD) of at least four independent experiments $(n=4)$. ${ }^{* *}$ indicates $p<0.001$ vs. untreated cells.

\subsection{Effect of Tobramycin on Tyrosinase Activity}

Melanin synthesis occurs in the melanosomes, and it is initiated with tyrosine oxidation by tyrosinase. Therefore, we measured the activity of tyrosinase, an enzyme important for melanin synthesis, in tobramycin-treated B16F10 cells. Tyrosinase activity was increased in tobramycin-treated cells compared to untreated cells (Figure 4). 


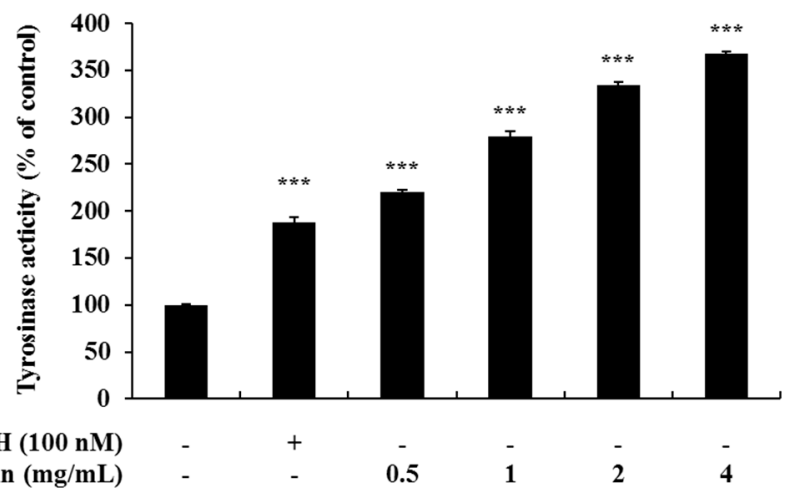

Figure 4. Tyrosinase activity in tobramycin-treated B16F10 melanoma cells. The cells were treated with various concentrations of tobramycin for $72 \mathrm{~h}$, and $\alpha$-MSH was used as a positive control. Data are presented as mean \pm standard deviation (SD) of at least four independent experiments $(n=4)$. *** indicates $p<0.001$ vs. untreated cells.

\subsection{Expression of Proteins Related to Melanin Synthesis}

\subsubsection{Tyrosinase, TRP-1, TRP-2}

Tyrosinase, TRP-1, and TRP-2 are important enzymes in melanin synthesis. Tobramycin stimulated melanin synthesis, and the effect of tobramycin on the intracellular expression of these three proteins was confirmed by Western blotting (Figure 5). Tyrosinase, TRP-1, and TRP-2 levels increased with higher tobramycin concentrations.

(a)

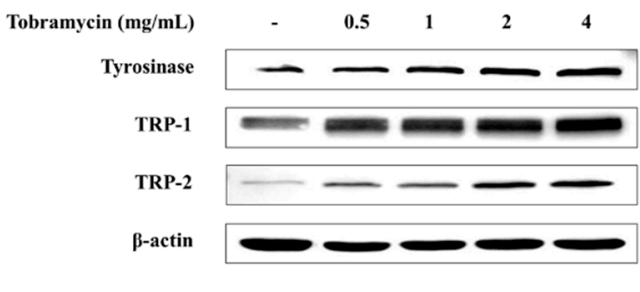

(c)

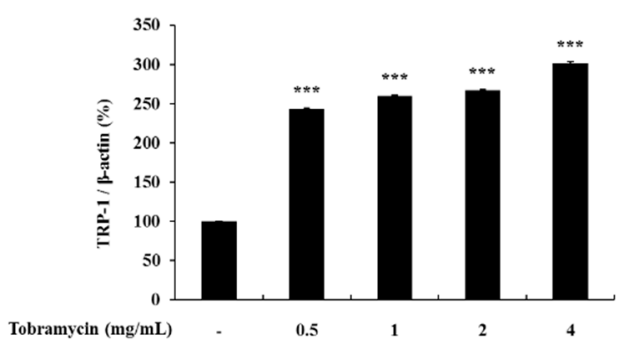

(b)

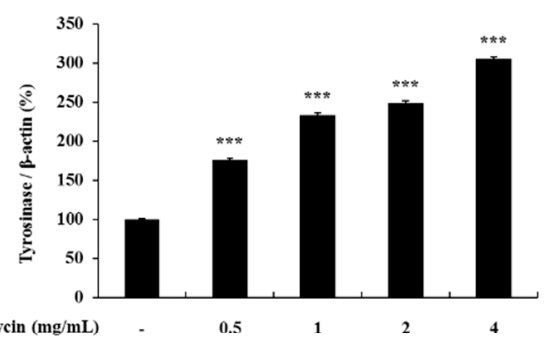

(d)

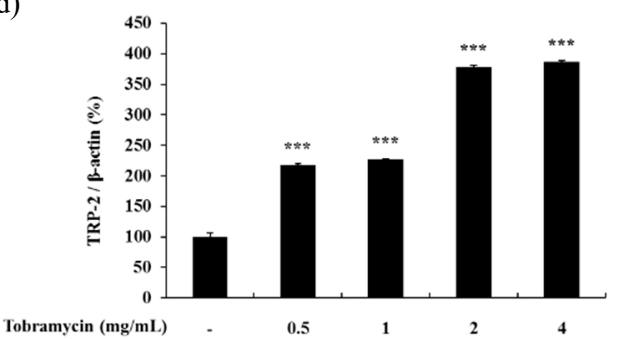

Figure 5. Effect of tobramycin on tyrosinase, TRP-1, and TRP-2 expression in B16F10 cells. Cells were treated with various concentrations of tobramycin for $40 \mathrm{~h}$. Protein levels were examined by Western blotting. (a) Representative Western blotting results and quantified (b) tyrosinase, (c) TRP-1, and (d) TRP-2 protein levels. Results are expressed as percentages of the control. Data are presented as mean \pm standard deviation (SD) of at least three independent experiments $(n=3)$. ${ }^{* * *}$ indicates $p<0.001$ vs. untreated cells.

\subsubsection{MITF}

MITF is a protein regulating tyrosinase, TRP-1, and TRP-2 expression. Therefore, the effect of tobramycin on MITF levels was evaluated. MITF expression increased in B16F10 cells treated with tobramycin for $20 \mathrm{~h}$ in a dose-dependent manner (Figure 6). These data imply that tobramycin increases MITF expression, which in turn upregulates tyrosinase, TRP-1, and TRP-2 levels. 
(a)

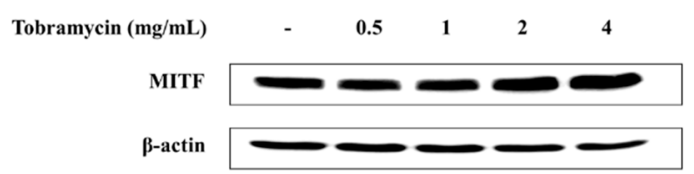

(b)

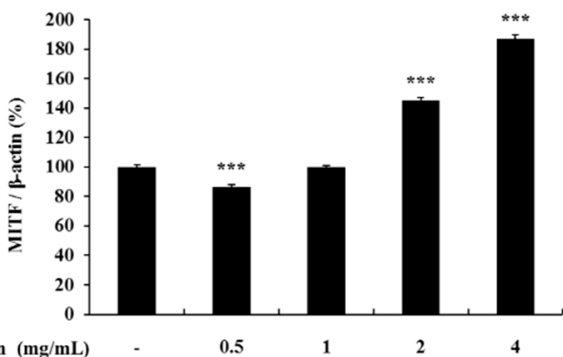

Figure 6. Effect of tobramycin on MITF expression in B16F10 cells. Cells were treated with various concentrations of tobramycin for $20 \mathrm{~h}$, and protein levels were examined by Western blotting. (a) Representative Western blotting results, and (b) quantified MITF protein levels. Results are expressed as percentages of the control. Data are presented as mean \pm standard deviation (SD) of at least three independent experiments $(n=3)$. ${ }^{* *}$ indicates $p<0.001$ vs. untreated cells.

\subsubsection{MAPKs and AKT}

The MAPK signaling pathway and the PI3K/AKT signaling pathway have been implicated in regulating MITF expression. MAPK family proteins, including ERK1/2, JNK, and p38, play an important role in melanin synthesis. Recent studies have shown that ERK phosphorylation degrades MITF by ubiquitination and inhibits TYR and TRP-1,2 expression, whereas JNK and p38 phosphorylation increases MITF expression, thereby inducing TYR and TRP-1,2 upregulation [26-28]. In addition, phosphorylated AKT has been implicated in melanin synthesis inhibition by phosphorylating MITF [29]. p38 phosphorylation levels tended to increase with higher concentrations of tobramycin in Western blot experiments. However, ERK, JNK, and AKT expression did not change in a dose-dependent manner (Figures 7 and 8).

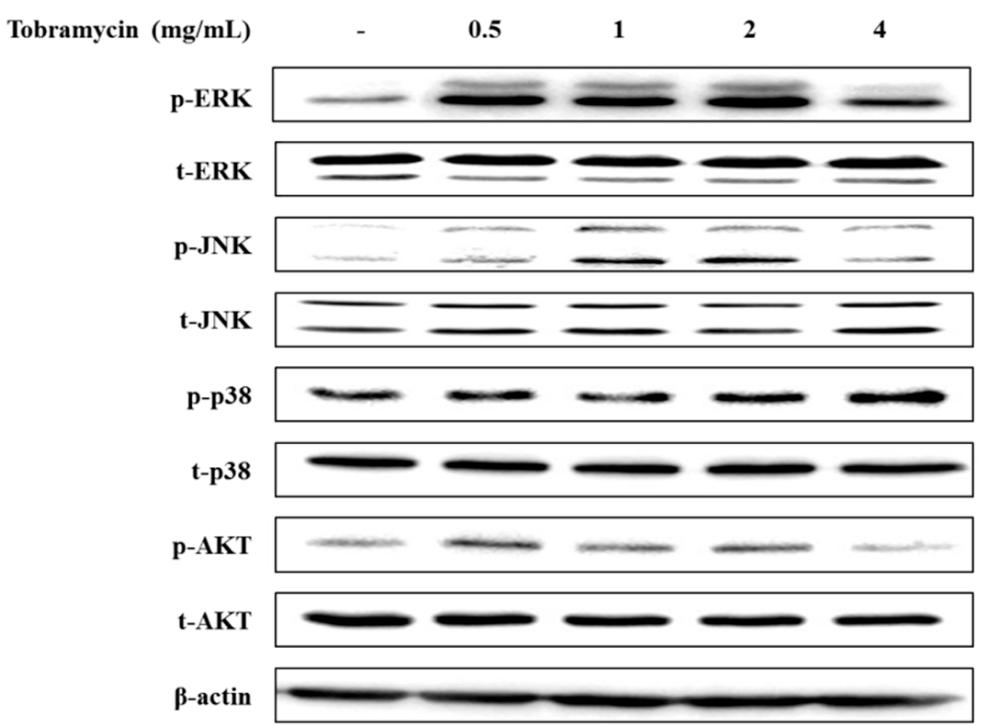

Figure 7. Effect of tobramycin on MAPK expression in B16F10 cells. Cells were treated with various concentrations of tobramycin for $4 \mathrm{~h}$. Protein levels were examined by Western blotting. 
(a)

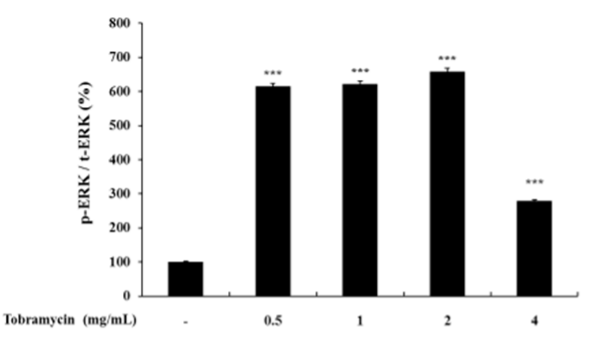

(b)

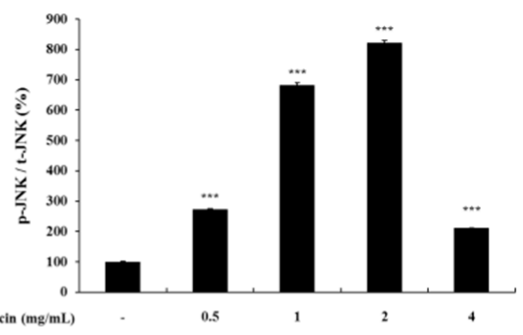

(c)
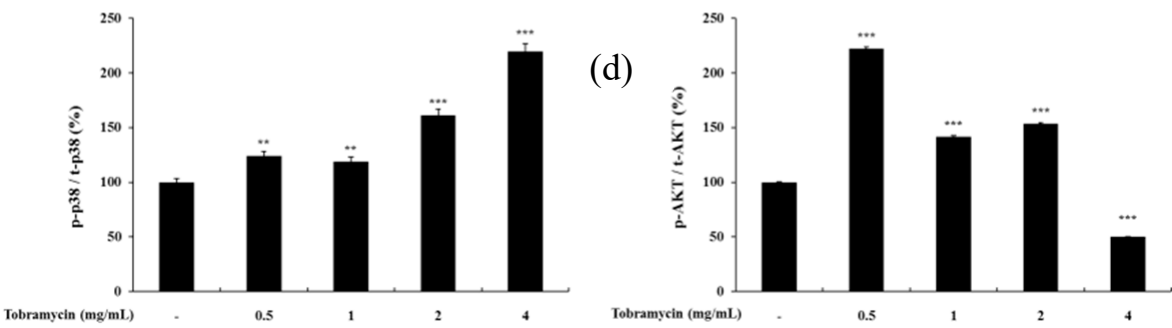

Figure 8. Quantified protein levels of (a) p-ERK, (b) p-JNK, (c) p-p38, and (d) p-AKT from Western blot experiments. Results are expressed as percentages of the control. Data are presented as mean $\pm \mathrm{SD}$ of at least three independent experiments $(n=3) .{ }^{* *}$ indicates $p<0.01,{ }^{* * *} p<0.001$ vs. control.

\subsection{Tyrosinase Activity Assay with Protein Inhibitors}

Tyrosinase activity increased when measured with an ERK inhibitor and a JNK inhibitor, but it decreased when a 38 inhibitor was used. These data confirm the notion that tobramycin phosphorylates p38, thereby increasing melanin formation (Figure 9). In addition, H89, a PKA inhibitor, was used to determine the effect of tobramycin on the cAMP/PKA signaling pathway (Figure 10). PKA activation increased MITF expression. Should tobramycin affect the cAMP/PKA signaling pathway, tyrosinase activity would be reduced using an PKA inhibitor; however, tyrosinase activity did not decrease with PKA inhibitor treatment. Tobramycin showed an increased cAMP/PKA signal. These data confirm that PKA is not involved in the effect of tobramycin on melanin synthesis.

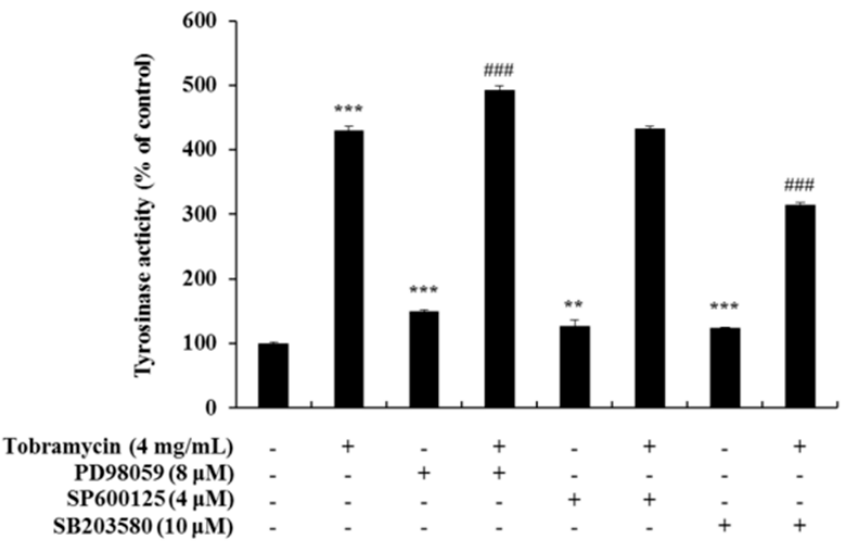

Figure 9. Effect of MAPK inhibitors on tobramycin-induced tyrosinase activity in B16F10 cells. To confirm the mechanism underlying the effect of tobramycin in melanogenesis, cellular tyrosinase activity was measured using the following MAPK inhibitors: PD98059 (ERK inhibitor), SP600125 (JNK inhibitor), and SB203580 (p38 inhibitor). Results are expressed as percentages of the control. Data are presented mean \pm SD of four independent experiments $(n=4)$. ${ }^{* *}$ indicates $p<0.01^{* * *}$ indicates $p<0.001$ vs. untreated cells and \#\#\# indicates $p<0.001$ vs. tobramycin treated cells. 


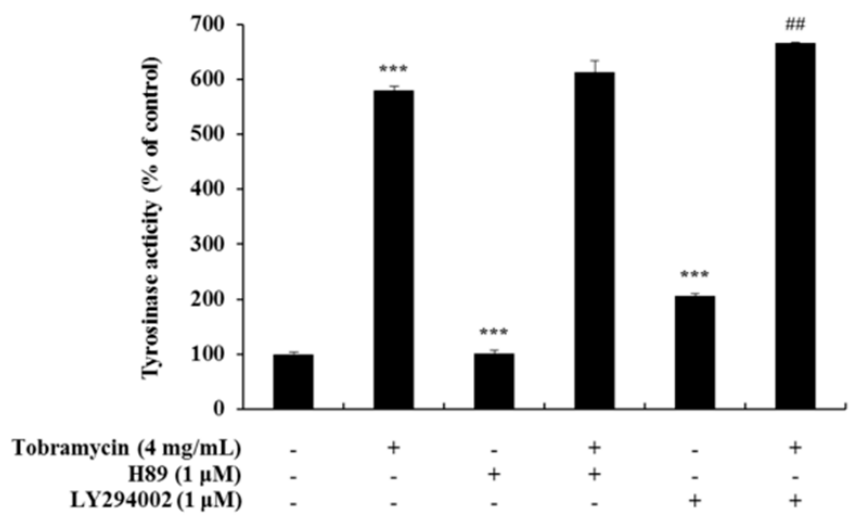

Figure 10. Effect of PKA inhibitors on tobramycin-induced tyrosinase activity in B16F10 cells. To confirm tobramycin mechanism in melanogenesis, cellular tyrosinase activity was measured using H89 (PKA inhibitor) and LY294002 (AKT inhibitor). Results are expressed as percentages of the control. Data are presented as mean \pm SD of four independent experiments $(n=4)$. ${ }^{* *}$ indicates $p<0.001$ vs. untreated cells and \#\# indicates $p<0.01$ vs. tobramycin treated cells.

\subsection{Effects of Tobramycin-Related Structures on Melanogenesis}

The cytotoxic effect of kanamycin A, kanamycin B, and 2-DOS, which share an antibiotic skeleton and similar structure with tobramycin, was measured (Figure 11). Subsequently, melanin content experiments were performed at concentration range showing no cytotoxicity. Kanamycin A demonstrated a tendency to decrease melanin content, whereas kanamycin B and 2-DOS did not change melanin content (Figures 12 and 13).

(a)

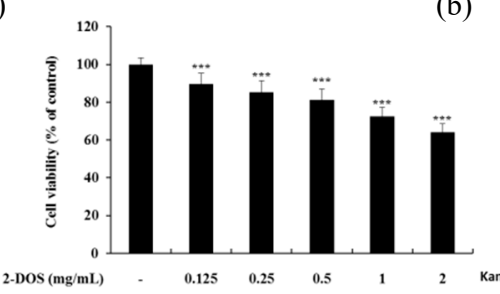

(b)

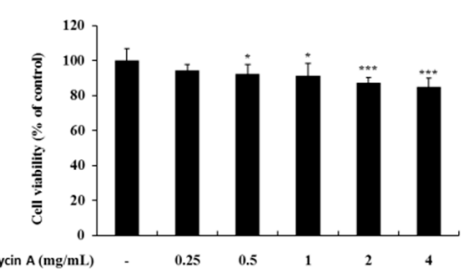

(c)

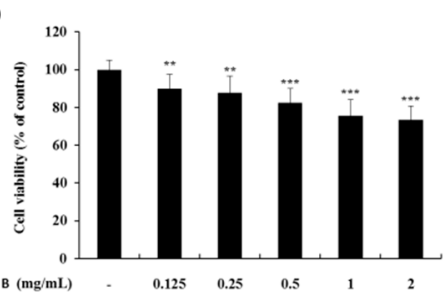

Figure 11. Cell viability of kanamycin A-(a), kanamycin B-(b), and 2-DOS-(c) treated B16F10 melanoma cells. Cells were treated with various concentrations of these drugs for $72 \mathrm{~h}$. Data are presented as mean \pm standard deviation (SD) of at least four independent experiments $(n=4) .{ }^{*}$ indicates $p<0.05$, ** $p<0.01,{ }^{* * *} p<0.001$ vs. untreated cells.

(a)

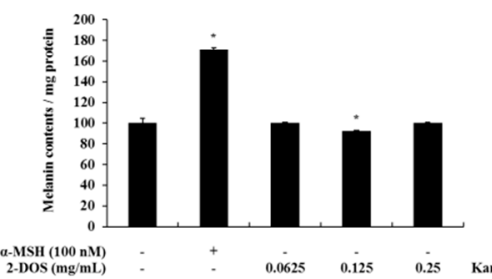

(b)

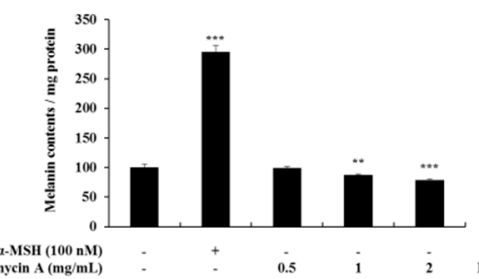

(c)

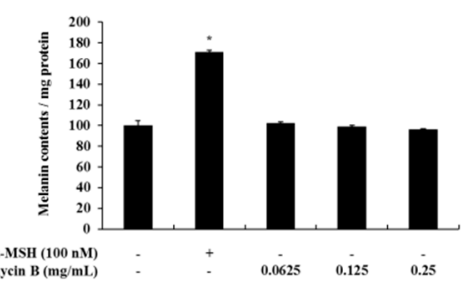

Figure 12. Melanin contents in 2-DOS-(a), kanamycin A-(b), and kanamycin B-(c) treated B16F10 melanoma cells. The cells were treated with various concentrations for $72 \mathrm{~h}$. $\alpha$-MSH was used as a positive control. Melanin concentrations are expressed as percentages compared to the respective values obtained for the control cells. Data are presented as mean \pm standard deviation (SD) of at least four independent experiments $(n=4)$. ${ }^{*}$ indicates $p<0.05,{ }^{* *} p<0.01,{ }^{* * *} p<0.001$ vs. untreated cells. 
(a)

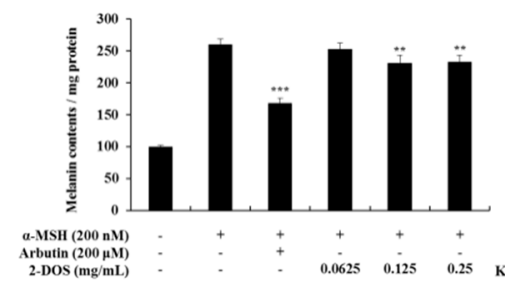

(b)

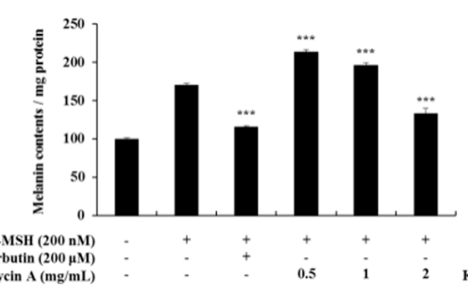

(c)

Figure 13. Melanin contents of 2-DOS(a), kanamycin A(b), and kanamycin B(c)-treated B16F10 melanoma cells. The cells were treated with various drug concentrations for $72 \mathrm{~h}$. $\alpha$-MSH was used as a negative control and arbutin was used as a positive control. Melanin concentrations are expressed as percentages compared to the respective values obtained for the control cells. Data are presented as mean \pm standard deviation (SD) of at least four independent experiments $(n=4)$. ${ }^{*}$ indicates $p<0.05$, ** $p<0.01,{ }^{* * *} p<0.001$ vs. tobramycin treatment.

\subsection{Cell Viability of HaCaT Keratinocyte Cells}

Both melanocytes and keratinocytes are present in the basal layer of the skin; furthermore, protrusions, extending from each melanocyte, are in contact with 30 to 40 peripheral keratinocytes. To confirm the effect of tobramycin on the keratinocytes surrounding melanocytes, an MTT assay was performed using a human cell line (HaCaT). Keratinocytes showed cell viability of $83 \%$ at $4 \mathrm{mg} / \mathrm{mL}$ tobramycin, in contrast to melanocytes, in which cell viability was significantly reduced at $8 \mathrm{mg} / \mathrm{mL}$ tobramycin (Figure 14).

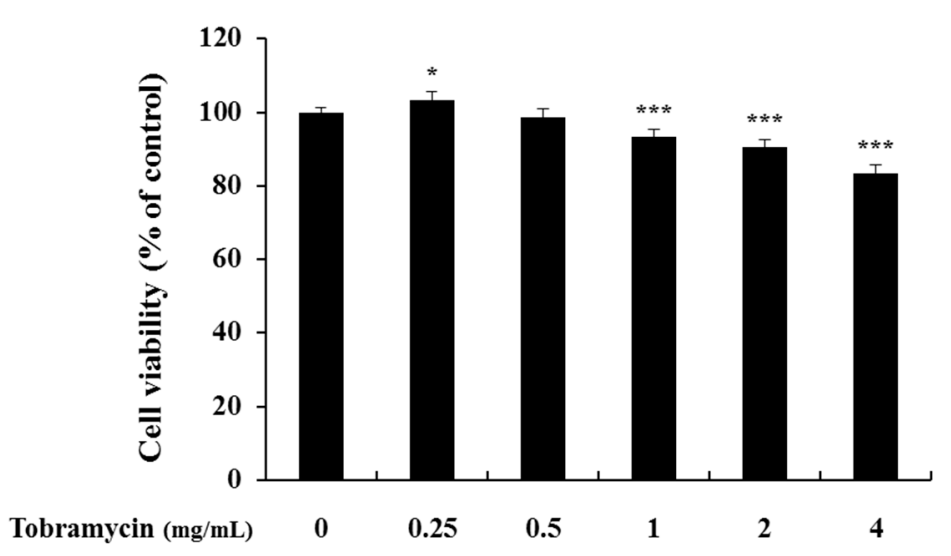

Figure 14. Cell viability of tobramycin-treated HaCaT keratinocyte cells. The cells were treated with various concentrations of tobramycin for $24 \mathrm{~h}$. Data are presented as mean \pm standard deviation (SD) of at least four independent experiments $(n=4)$. ${ }^{*}$ indicates $p<0.05,{ }^{* * *} p<0.001$ vs. control.

\section{Discussion}

Melanin, which protects the skin against UVR, is produced in melanosomes and is present in the human skin, hair, eyes, ears, and even brain [30,31]. Excessive pigmentation causes hyperpigmentation, whereas insufficient pigmentation is associated with the degradation of pigmentation [6]. Here, we studied the effect of tobramycin, one of the aminoglycoside antibiotics, on melanogenesis. Tobramycin has been in clinical use for a long time; however, it has demonstrated several side effects and has increasingly been replaced by improved antibiotics. Here, we demonstrated the tobramycin effect on melanin formation in B16F10 cells. First, a MTT assay was performed to evaluate tobramycin cytotoxicity. It confirmed that tobramycin treatment increases the activity of tyrosinase, which is the most important enzyme for melanin synthesis. Furthermore, tobramycin upregulated melanin levels in a concentration-dependent manner at a concentration range which did not cause cytotoxicity. We identified the effects of tobramycin on the cAMP/PKA, MAPK, and PI3K/AKT signaling pathways, which are important pathways for melanogenesis, using Western blot experiments and the tyrosinase 
activity assay with protein inhibitors. First, the expression levels of tyrosinase, TRP-1, and TRP-2, the most relevant enzymes for melanin synthesis, were increased when cells were treated with tobramycin. MITF is a factor known for controlling the levels of these three enzymes; its expression was increased in B16F10 cells treated with tobramycin for $20 \mathrm{~h}$ in a dose-dependent manner. The mechanism involved in MITF expression included tobramycin phosphorylation of the p38 protein, one of the MAPK proteins, among various signal transduction pathways. Phosphorylated p38 increased MITF expression, which in turn upregulated tyrosinase, TRP-1, and TRP-2 levels, leading to heightened melanin synthesis.

The structure of the aminoglycoside antibiotics includes a central skeleton, which is an aminocyclitol ring with an amino sugar bound to it. Kanamycin A, kanamycin B, and tobramycin incorporate the central structure presented by 2-DOS. Furthermore, kanamycin A, kanamycin B, and tobramycin contain a structure in which an amino sugar is bonded to carbons 4 and 6 . The effect of the basic skeleton structure, 2-DOS, on melanin synthesis (Figures 11 and 12) was measured in a concentration range not causing cytotoxicity (Figure 11). Treatment with 2-DOS did not affect melanogenesis, suggesting that the 2-DOS structure does not influence tobramycin-induced melanogenesis. Tobramycin is a derivative of kanamycin, which has a very similar structure. Kanamycin A, kanamycin B, and tobramycin decreased, did not influence, and improved melanin production, respectively [32]. Structural comparison of the three drugs shows that the structure of the amino sugar bound to the 4th carbon of 2-DOS is slightly different. Kanamycin A binds to 2'-OH, 3'-OH, 4'-OH, 5'-CH2NH2, whereas kanamycin B binds to 2'-NH2, 3'-OH, 4'-OH, 5'-CH2NH2, and tobramycin binds to $2^{\prime}-\mathrm{NH} 2,4^{\prime}-\mathrm{OH}, 5^{\prime}-\mathrm{CH} 2 \mathrm{NH} 2$ (Figure 15). The data imply that tobramycin effect on melanogenesis is due to the structural difference of the amino sugars bound to the 4-carbon of 2-DOS.

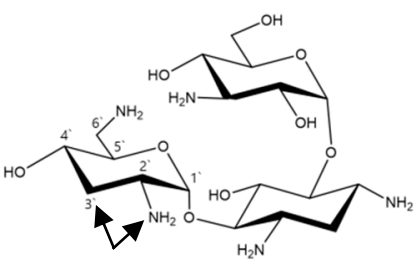

Tobramycin

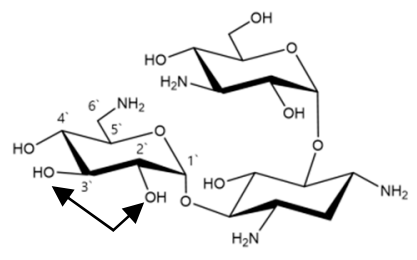

Kanamycin A

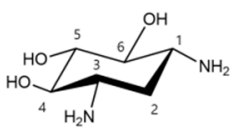

2-DOS

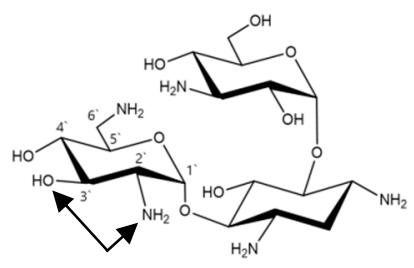

Kanamycin B

Figure 15. Structures of 2-DOS, tobramycin, kanamycin A, and kanamycin B.

Melanocytes are uniformly distributed throughout the basal layer of the skin epidermis [33]. Melanin, produced from melanocytes, migrates to surrounding keratinocytes. Due to the close relationship between melanocytes and keratinocytes, tobramycin cytotoxicity was evaluated on keratinocytes (Figure 12). The cytotoxicity of tobramycin in HaCaT human keratinocytes was $83 \%$ and more than $90 \%$ after $4 \mathrm{mg} / \mathrm{mL}$ and $2 \mathrm{mg} / \mathrm{mL}$ tobramycin treatment, respectively. Even though tobramycin demonstrated slight toxicity at $4 \mathrm{mg} / \mathrm{mL}$ in HaCaT human keratinocytes, it could be used at lower concentrations. We have confirmed from the above experiments that Tobramycin has the effect of improving melanin synthesis in melanocytes, apart from the role of antibiotics. Additional experiments will be needed for medical use but we believe this has proven the new efficacy of this old antibiotic, which is no longer in use. 


\section{Materials and Methods}

\subsection{Chemicals and Reagents}

Tobramycin and kanamycin A were purchased from Tokyo Chemical Industry Co., Ltd. (Chuo-ku, Tokyo, Japan). Dulbecco's Modified Eagle Medium (DMEM), fetal bovine serum (FBS), penicillin/streptomycin, trypsin-ethylenediaminetetraacetic acid, PD98059, and BCA kit were procured from Thermo Fisher Scientific (Waltham, MA, USA). $\alpha-\mathrm{MSH}, \mathrm{NaOH}, \mathrm{MTT}$, and kanamycin B were obtained from Sigma-Aldrich (St. Louis, MO, USA). Antibodies against tyrosinase, TRP-1, TRP-2, and MITF were obtained from Santa Cruz Biotechnology (Dallas, TX, USA). Antibodies against p-p38, p38, p-JNK, JNK, p-ERK, ERK, p-AKT, AKT, and $\beta$-actin were purchased from Cell Signaling Technology (Danvers, MA, USA). Radioimmunoprecipitation assay (RIPA) buffer, dimethyl sulfoxide (DMSO), enhanced chemiluminescence (ECL) kit, and 2× Laemmli sample buffer were obtained from Biosesang (Sungnam, Gyeonggi-do, Korea). SP600125 and SB203580 were purchased from Cayman Chemical (Ann Arbor, MI, USA) and Calbiochem (San Diego, CA, USA); 2-DOS was obtained from Toronto Research Chemicals Inc. (Toronto, Ontario, Canada). EZ-LDH cell cytotoxicity assay kit was purchased from DoGenBio Co.,Ltd (Guro, Seoul, Korea).

\subsection{Cell Culture}

B16F10 murine melanoma cells and HaCaT keratinocyte cells were cultured in DMEM medium supplemented with $10 \%$ heat inactivated FBS and $1 \%$ penicillin/streptomycin at $37^{\circ} \mathrm{C}$ in a humidified atmosphere containing $5 \% \mathrm{CO}_{2}$.

\subsection{Cell Viability Assay}

Cell viability was determined by MTT assay. B16F10 melanoma cells $\left(3.0 \times 10^{4}\right.$ cells/well) and HaCaT keratinocyte cells $\left(1 \times 10^{4}\right.$ cells/well $)$ were seeded into 24 -well plates and incubated for $24 \mathrm{~h}$ in culture medium. The cells were treated with various concentrations of tobramycin $(0.5,1,2,4,8$, and $16 \mathrm{mg} / \mathrm{mL})$, kanamycin A $(0.25,0.5,1,2$, and $4 \mathrm{mg} / \mathrm{mL})$, kanamycin B $(0.125,0.25,0.5,1$, and $2 \mathrm{mg} / \mathrm{mL})$, and 2-DOS $(0.125,0.25,0.5,1$, and $2 \mathrm{mg} / \mathrm{mL})$ for $24 \mathrm{~h}$ (HaCaT) or $72 \mathrm{~h}$ (B16F10). After incubation, MTT solution was added to a final concentration of $0.2 \mathrm{mg} / \mathrm{mL}$ for $2 \mathrm{~h}$. Next, the MTT solution was removed, the produced formazan crystals were dissolved by DMSO, and absorbance was measured at $540 \mathrm{~nm}$ on a spectrophotometric microplate reader (Tecan, Mannedorf, Switzerland).

\subsection{Lactate Dehydrogenase (LDH) Release Assay}

B16F10 cells were seeded in a 24-well plate and cultured $24 \mathrm{~h}$ and incubated with various concentrations of tobramycin $(8$ and $16 \mathrm{mg} / \mathrm{mL}$ ) for $72 \mathrm{~h}$. After incubation, $10 \mu \mathrm{L}$ of the culture supernatant was transferred into a new 96-well plate. LDH released to the extracellular medium was quantified by the EZ-LDH cell cytotoxicity assay kit according to the manufacturer's instructions. Fresh culture medium was used as blank. The absorbance was measured at $450 \mathrm{~nm}$ using an ELISA reader.

\subsection{Melanin Content Measurement}

B16F10 cells $\left(1.0 \times 10^{5}\right.$ cells/well $)$ were seeded into $60 \pi$ dish and incubated for $24 \mathrm{~h}$ in culture medium. Next, the medium was removed, and the cells were treated with various tobramycin $(0.5,1,2$, and $4 \mathrm{mg} / \mathrm{mL})$, kanamycin $\mathrm{A}(0.5,1$, and $2 \mathrm{mg} / \mathrm{mL})$, kanamycin B $(0.0625,0.125$, and $0.25 \mathrm{mg} / \mathrm{mL})$, and 2-DOS $(0.0625,0.125,0.25 \mathrm{mg} / \mathrm{mL})$ concentrations, $100 \mathrm{nM}$ or $200 \mathrm{nM} \alpha-\mathrm{MSH}$, and $200 \mathrm{nM}$ arbutin for $72 \mathrm{~h}$. After incubation, the cells were washed with PBS once, and were dissolved in $2 \mathrm{~mL} 1 \mathrm{~N} \mathrm{NaOH}$ containing $10 \% \mathrm{DMSO}$ at $70{ }^{\circ} \mathrm{C}$ for $1 \mathrm{~h}$. Then, $200 \mu \mathrm{L} \mathrm{NaOH}$ solution aliquots were transferred to 96-well plates, and absorbance was measured at $405 \mathrm{~nm}$ on a spectrophotometric microplate reader (Tecan, Mannedorf, Switzerland). The $\mathrm{NaOH}$ solution protein concentration was quantified by a bicinchoninic acid (BCA) protein assay kit. 


\subsection{Intracellular Tyrosinase Activity}

B16F10 cells $\left(1.0 \times 10^{5}\right.$ cells/well $)$ were seeded into a $60 \pi$ dish and incubated for $24 \mathrm{~h}$ in culture medium. Next, the medium was removed, and the cells were treated with various tobramycin $(0.5,1$, 2 , and $4 \mathrm{mg} / \mathrm{mL})$, kanamycin A $(0.5,1$, and $2 \mathrm{mg} / \mathrm{mL})$, kanamycin B $(0.0625,0.125$, and $0.25 \mathrm{mg} / \mathrm{mL})$, and 2-DOS $(0.0625,0.125$, and $0.25 \mathrm{mg} / \mathrm{mL})$ concentrations, $100 \mathrm{nM}$ or $200 \mathrm{nM} \alpha-\mathrm{MSH}, 200 \mathrm{nM}$ arbutin, and protein inhibitors for $72 \mathrm{~h}$. After incubation, the cells were washed with PBS once, and were treated with RIPA buffer (supplemented with $1 \%$ protease inhibitor cocktail) for $20 \mathrm{~min}$ at $4{ }^{\circ} \mathrm{C}$. Cell lysates were collected with cell scrapers and transferred to $1.5 \mathrm{~mL}$ microtubes. Collected lysates were centrifuged at $15,000 \times g$ for $25 \mathrm{~min}$, and the supernatant was separated. Protein concentration was quantified by BCA protein assay kit. The amount of quantified protein in the samples was equalized using $0.1 \mathrm{M}$ sodium phosphate buffer; the samples were then mixed with $2 \mathrm{mg} / \mathrm{mL}$ L-DOPA per well in 96-well plates. After incubation at $37^{\circ} \mathrm{C}$ and centrifugation at $300 \mathrm{rpm}$ for $1 \mathrm{~h}$, absorbance was measured at $490 \mathrm{~nm}$ on a spectrophotometric microplate reader.

\subsection{Western Blot Analysis}

B16F10 cells $\left(1.0 \times 10^{5}\right.$ cells/dish $)$ were seeded into a $60 \pi$ dish, incubated, and then treated with various concentrations of tobramycin $(0.5,1,2$, and $4 \mathrm{mg} / \mathrm{mL})$ for predetermined periods of time to analyze the expression of proteins of interest. After incubation, the cells were collected and lysed in RIPA buffer (supplemented 1\% protease inhibitor cocktail). Next, collected lysates were centrifuged at $15,000 \times g$ for $25 \mathrm{~min}$, and the supernatant was separated. Protein concentration in the supernatant was quantified by BCA protein assay kit. Supernatant and 5x SDS-PAGE buffer (4:1) were mixed to prepare Western blotting samples (protein concentration: $40 \mu \mathrm{g})$. Finally, samples $(10 \mu \mathrm{L})$ were loaded on sodium dodecyl sulfate-polyacrylamide gels.

\subsection{Statistical Analysis}

The results of the experiments were analyzed using Student's $t$-test. $p$-values $<0.05\left(^{*}\right)$ or $0.01(* *, \#)$ or $0.001(* * *, \# \#)$ were considered statistically significant and all data are expressed as percentages compared to the respective values of the control or negative control. All data has been expressed as mean $\pm \mathrm{SD}$ of at least three independent experiments.

Author Contributions: Conceptualization, C.-G.H.; methodology, Y.C.C. and S.-H.M.; project administration, C.-G.H.; validation, S.-H.M.; formal analysis, S.-H.M.; investigation, S.-H.M.; data curation, S.-H.M.; writing-original draft preparation, C.-G.H. and S.-H.M.; writing-review and editing, C.-G.H., Y.C.C. and S.-H.M.; supervision, C.-G.H.

Funding: This research received no external funding.

Acknowledgments: This research was supported by the 2018 Scientific Promotion Program funded by Jeju National University, South Korea.

Conflicts of Interest: The authors declare no conflict of interest.

\section{References}

1. Brenner, M.; Hearing, V.J. The protective role of melanin against UV damage in human skin. Photochem. Photobiol. 2008, 84, 539-549. [CrossRef]

2. D'Orazio, J.; Jarrett, S.; Amaro-Ortiz, A.; Scott, T. UV radiation and the skin. Int. J. Mol. Sci. 2013, 14, 12222-12248. [CrossRef]

3. Tran, T.N.T.; Schulman, J.; Fisher, D.E. UV and pigmentation: Molecular mechanisms and social controversies. Pigment Cell Melanoma Res. 2008, 21, 509-516. [CrossRef]

4. Pillaiyar, T.; Manickam, M.; Jung, S.-H. Recent development of signaling pathways inhibitors of melanogenesis. Cell. Signal. 2017, 40, 99-115. [CrossRef]

5. Pillaiyar, T.; Manickam, M.; Jung, S.H. Inhibitors of melanogenesis: A patent review (2009-2014). Expert Opin. Ther. Pat. 2015, 25, 775-788. [CrossRef] 
6. Aroca, P.; Urabe, K.; Kobayashi, T.; Tsukamoto, K.; Hearing, V.J. Melanin biosynthesis patterns following hormonal stimulation. J. Biol. Chem. 1993, 268, 25650-25655.

7. Slominski, A.; Wortsman, J.; Plonka, P.M.; Schallreuter, K.U.; Paus, R.; Tobin, D.J. Hair follicle pigmentation. J. Investig. Dermatol. 2005, 124, 13-21. [CrossRef]

8. Imesch, P.D.; Wallow, I.H.; Albert, D.M. The color of the human eye: A review of morphologic correlates and of some conditions that affect iridial pigmentation. Surv. Ophthalmol. 1997, 41 (Suppl. 2), S117-S123. [CrossRef]

9. Kaidbey, K.H.; Agin, P.P.; Sayre, R.M.; Kligman, A.M. Photoprotection by melanin-A comparison of black and Caucasian skin. J. Am. Acad. Dermatol. 1979, 1, 249-260. [CrossRef]

10. Barr, A.J. The biochemical basis of disease. Essays Biochem. 2018, 62, 619-642. [CrossRef]

11. Franks, A.G., Jr. Skin manifestations of internal disease. Med. Clin. N. Am. 2009, 93, 1265-1282. [CrossRef]

12. Pinto, F.J.; Bolognia, J.L. Disorders of hypopigmentation in children. Pediatr. Clin. N. Am. 1991, 38, 991-1017. [CrossRef]

13. Brogden, R.; Pinder, R.; Sawyer, P.R.; Speight, T.; Avery, G. Tobramycin: A review of its antibacterial and pharmacokinetic properties and therapeutic use. Drugs 1976, 12, 166-200. [CrossRef]

14. Kharel, M.K.; Basnet, D.B.; Lee, H.C.; Liou, K.; Woo, J.S.; Kim, B.G.; Sohng, J.K. Isolation and characterization of the tobramycin biosynthetic gene cluster from Streptomyces tenebrarius. FEMS Microbiol. Lett. 2004, 230, 185-190. [CrossRef]

15. Neu, H.C. Tobramycin: An overview. J. Infect. Dis. 1976, 134, S3-S19. [CrossRef]

16. Higgins, C.E.; Kastner, R.E. Nebramycin, a new broad-spectrum antibiotic complex. II. Description of Streptomyces tenebrarius. Antimicrob. Agents Chemother. 1967, 7, 324-331.

17. Stark, W.M.; Hoehn, M.M.; Knox, N.G. Nebramycin, a new broad-spectrum antibiotic complex. I. Detection and biosynthesis. Antimicrob. Agents Chemother. 1967, 7, 314-323.

18. Thompson, R.Q.; Presti, E.A. Nebramycin, a new broad-spectrum antibiotic complex. 3. Isolation and chemical-physical properties. Antimicrob. Agents Chemother. 1967, 7, 332-340.

19. Wick, W.E.; Welles, J.S. Nebramycin, a new broad-spectrum antibiotic complex. IV. In vitro and in vivo laboratory evaluation. Antimicrob. Agents Chemother. 1967, 7, 341-348.

20. Cannella, C.A.; Wilkinson, S.T. Acute renal failure associated with inhaled tobramycin. Am. J. Health-Syst. Pharm. 2006, 63, 1858-1861. [CrossRef]

21. Gonzalez-Mendiola, M.R.; Balda, A.G.; Delgado, M.C.; Montano, P.P.; De Olano, D.G.; Sanchez-Cano, M. Contact allergy from tobramycin eyedrops. Allergy 2005, 60, 527-528. [CrossRef]

22. Neu, H.C.; Bendush, C.L. Ototoxicity of tobramycin: A clinical overview. J. Infect. Dis. 1976, 134, S206-S218. [CrossRef]

23. Daschner, F.D.; Just, H.M.; Jansen, W.; Lorber, R. Netilmicin versus tobramycin in multi-centre studies. J. Antimicrob. Chemother. 1984, 13 (Suppl. A), 37-45. [CrossRef]

24. Moellering, R.C., Jr.; Wennersten, C.; Kunz, L.J.; Poitras, J.W. Resistance to gentamicin, tobramycin and amikacin among clinical isolates of bacteria. Am. J. Med. 1977, 62, 873-881. [CrossRef]

25. Barclay, M.L.; Begg, E.J.; Chambers, S.T.; Thornley, P.E.; Pattemore, P.K.; Grimwood, K. Adaptive resistance to tobramycin in Pseudomonas aeruginosa lung infection in cystic fibrosis. J. Antimicrob. Chemother. 1996, 37, 1155-1164. [CrossRef]

26. Chung, Y.C.; Kim, S.; Kim, J.H.; Lee, G.S.; Lee, J.N.; Lee, N.H.; Hyun, C.-G. Pratol, an O-methylated flavone, induces melanogenesis in B16F10 melanoma cells via p-p38 and p-jnk upregulation. Molecules 2017, 22, 1704. [CrossRef]

27. Ko, G.-A.; Cho, S.K. Phytol suppresses melanogenesis through proteasomal degradation of MITF via the ROS-ERK signaling pathway. Chem.-Biol. Interact. 2018, 286, 132-140. [CrossRef]

28. Ye, Y.; Wang, H.; Chu, J.H.; Chou, G.X.; Yu, Z.L. Activation of p38 MAPK pathway contributes to the melanogenic property of apigenin in B16 cells. Exp. Dermatol. 2011, 20, 755-757. [CrossRef]

29. Su, T.R.; Lin, J.J.; Tsai, C.C.; Huang, T.K.; Yang, Z.Y.; Wu, M.O.; Zheng, Y.Q.; Su, C.C.; Wu, Y.J. Inhibition of melanogenesis by gallic acid: Possible involvement of the PI3K/Akt, MEK/ERK and Wnt/beta-catenin signaling pathways in B16F10 cells. Int. J. Mol. Sci. 2013, 14, 20443-20458. [CrossRef]

30. Gratacap, B.; Attard, A.; Laurent, A.; Stoebner, P.; Smirou, D.; Charachon, R. Melanin in the inner ear. An experimental study with control and kanamycin-intoxicated colored guinea-pigs. Arch. Oto-Rhino-Laryngol. 1989, 246, 235-237. [CrossRef] 
31. Zecca, L.; Bellei, C.; Costi, P.; Albertini, A.; Monzani, E.; Casella, L.; Gallorini, M.; Bergamaschi, L.; Moscatelli, A.; Turro, N.J.; et al. New melanic pigments in the human brain that accumulate in aging and block environmental toxic metals. Proc. Natl. Acad. Sci. USA 2008, 105, 17567-17572. [CrossRef]

32. Wrzesniok, D.; Otreba, M.; Beberok, A.; Buszman, E. Impact of kanamycin on melanogenesis and antioxidant enzymes activity in melanocytes-An in vitro study. J. Cell. Biochem. 2013, 114, 2746-2752. [CrossRef]

33. Thingnes, J.; Lavelle, T.J.; Hovig, E.; Omholt, S.W. Understanding the melanocyte distribution in human epidermis: An agent-based computational model approach. PLoS ONE 2012, 7, e40377. [CrossRef] 